\title{
Phase-dependent atomic ionization in few-cycle intense laser fields
}

\author{
X. M. Tong, ${ }^{1,2, *}$ K. Hino, ${ }^{1,2}$ and N. Toshima ${ }^{1}$ \\ ${ }^{1}$ Institute of Materials Science, Graduate School of Pure and Applied Sciences, University of Tsukuba, 1-1-1 Tennodai, Tsukuba, Ibaraki \\ 305-8573, Japan \\ ${ }^{2}$ Center for Computational Sciences, University of Tsukuba, 1-1-1 Tennodai, Tsukuba, Ibaraki 305-8577, Japan
}

(Received 17 May 2006; published 28 September 2006)

\begin{abstract}
We have studied the carrier-envelope phase-dependent above-threshold-ionization processes by solving the time-dependent Schrödinger equation nonperturbatively. Our simulated carrier-envelope phase-dependent above-threshold-ionization spectra are in good agreement with the experimental measurement of Paulus et al. [Phys. Rev. Lett. 91, 253004 (2003)] if we shift the experimental carrier-envelope phase by $0.15 \pi$. This means that we have recalibrated the carrier-envelope phase more accurately by comparing our simulations with the experimental measurements. Furthermore, by tracing back the origin of the ionized electrons, we have clearly identified that the low-energy electrons come from the direct ionization by the laser field and the high-energy electrons come from the recollision with the parent core after being bounced back by the laser field.
\end{abstract}

DOI: 10.1103/PhysRevA.74.031405

PACS number(s): $32.80 . \mathrm{Rm}, 42.50 . \mathrm{Hz}, 42.65 . \mathrm{Re}$

Interaction of atoms with an intense laser field has been studied extensively in the past two decades. The two major processes, high-order harmonic generation (HHG) $[1,2]$ and above-threshold ionization (ATI) [3], can be described by the rescattering model $[4,5]$ qualitatively. The laser-atom interactions involve three steps: (a) an electron is ionized through tunneling ionization when the laser field reaches the peak; (b) then the tunneling electron travels out (direct ionization) or is bounced back by the laser field when the field changes its direction (rescattering); and (c) the rescattered electron recombines with the parent core by emitting a photon (HHG) or is scattered by the parent core (high-energy ATI). Based on this concept, Lewenstein et al. [6] developed a formula using the strong field approximation (SFA), which neglects the electron-parent-core interaction after the tunneling ionization. Generally speaking, one can study the ATI spectra by solving the time-dependent Schrödinger equation [7-10] or by the $S$-matrix method with the SFA [11-13]. The former gives more reasonable photoelectron energy spectra in the low-energy regime, while the latter provides better physical pictures. Although the rescattering model provides an intuitive picture of atom-laser interactions, experimentally it is impossible to probe the classical trajectory directly.

Advances in laser technology have made it possible to produce few-cycle laser pulses, and the interaction of atoms with ultrashort intense laser has become of current research interest [14-18]. Such a short laser pulse enables us to study the ionization process in detail by decoupling the tunneling ionization and the interference effect. Moreover, the motion of the ionized electron can be controlled by the timedependent laser field. Based on the SFA, Salières et al. [19] found that a few classical trajectories are responsible for explaining the ATI spectra in an elliptical laser field. In an ultrashort intense laser field, the ATI spectra are very sensitive to the details of the time-dependent electric field rather than the laser frequency. For short laser pulses, the electric field is expressed as $\mathbf{E}(t)=\mathbf{E}_{0} f(t) \cos (\omega t+\delta)$ with $\left|\mathbf{E}_{0}\right|$ the peak field, $f(t)$ the field envelope, $\omega$ the laser frequency, and $\delta$ the carrier-envelope (CE) phase. The CE phase describes the offset of the peak laser field relative to the peak position of the envelope. By tuning the $\mathrm{CE}$ phase, one can steer atomic processes or modify ionized electron wave packets. Such an electron wave packet can be potentially used to image molecular structures $[20,21]$. Profound knowledge of the CE phase is highly desired for these studies. Although the CE phase can be stabilized and tuned in experiments [22,23], direct measurement of the CE phase is very difficult so far. Usually the CE phase is extracted by the interaction of fewcycle laser pulses with atoms or materials. The CE phase obtained in this way depends strongly on the reliability of the theoretical models. The CE phase has been studied by electron ionization from the metal surface $[23,24]$ and by highenergy ATI spectra in ultrashort laser pulses $[22,25]$. The measured CE phase-dependent ionizations [23] from the metal surface agreed with the simulation [24] qualitatively. Although the ATI spectra measured by Paulus et al. [22] and simulated by the $S$-matrix method of Milosevic et al. [26] are in agreement qualitatively, some quantitative discrepancies still exist. Since one relies on the simulation to extract the CE phase from the experiment, more accurate theoretical studies are greatly needed. Encouraged by these developments, we have developed a full quantum nonperturbative time-dependent scheme that keeps the intuitive picture of the $S$-matrix method [13] without recourse to the SFA.

In our present simulation scheme, instead of solving the time-dependent Scrödinger equation in the differential form, we propagate the time-dependent wave function in the integral form from time $T=-\infty$ to $t$ as (atomic units are used)

$$
\Psi(t)=-i \int_{T}^{t} U(t, \tau) V U_{0}(\tau, T) \Phi_{0} d \tau+U_{0}(t, T) \Phi_{0},
$$

with

$$
U_{0}(t, T)=e^{-i H_{0}(t-T)} \quad \text { and } \quad U(t, T)=\exp \left(-i \int_{T}^{t} H d t^{\prime}\right)
$$

\footnotetext{
*Corresponding author. Electronic address: tong@ims.tsukuba.ac.jp
}

Here, $H_{0}$ is the atomic Hamiltonian, $V=-\mathbf{r} \cdot \mathbf{E}(t)$ is the timedependent laser-atom interaction, with $\mathbf{r}$ denoting the elec- 


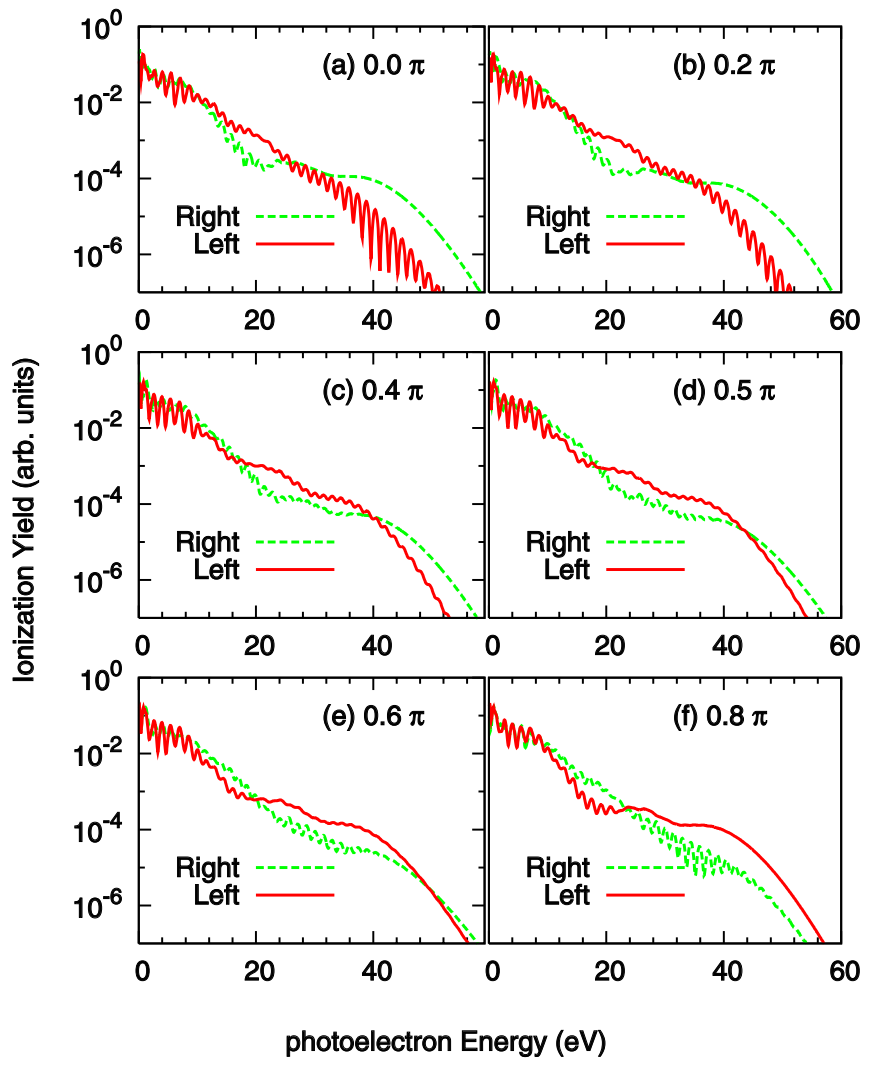

FIG. 1. (Color online) Ionization yields to the right and left sides as a function of the CE phase. The laser intensity is 6 $\times 10^{13} \mathrm{~W} / \mathrm{cm}^{2}$ and laser pulse duration is $6 \mathrm{fs}$ with central wavelength of $800 \mathrm{~nm}$.

tron position, $H=H_{0}+V$, and $\Phi_{0}$ is the initial field-free wave function. The first term in Eq. (1) shows that the initial wave function $\Phi_{0}$ propagates to time $\tau$ under $H_{0}$, and interacts with the laser field through $V$ at time $\tau$ (ionization) and then propagates to time $t$ under the full Hamiltonian. The second term indicates that the initial wave function $\Phi_{0}$ propagates to time $t$ under $H_{0}$ and it does not contribute to any dynamic processes. If we replace $U(t, T)$ by

$$
U_{v}(t, T)=\exp \left(-i \int_{T}^{t}\left[\mathbf{p}-\mathbf{A}\left(t^{\prime}\right)\right]^{2} / 2 d t^{\prime}\right)
$$

in Eq. (1) with $\mathbf{p}$ the momentum of the ionized electron and

$$
\mathbf{A}\left(t^{\prime}\right)=\int_{t^{\prime}}^{\infty} \mathbf{E}(t) d t
$$

we will get back to the Lewenstein model [6]. In Eq. (1), we separate the field-free wave function from the total wave function. The advantages of this separation are (a) that for a relatively weak field, the background wave function is factored out so that the numerical accuracy can be improved; and (b) that the creation and propagation processes are separated so that we can illustrate the physics more clearly.

In the practical calculation, we propagate the wave function in the full Hamiltonian by a generalized pseudospectral method in the energy domain [27,28]. The effectiveness of the method has been shown in the studies of HHG [28], ATI

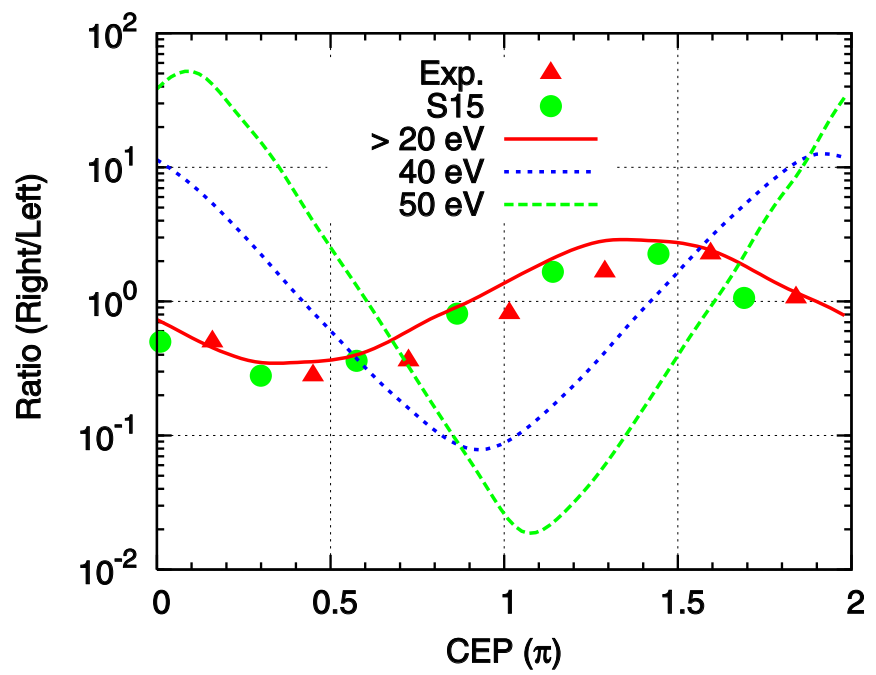

FIG. 2. (Color online) Ratio of the ionization yields from the right and left sides for different photoelectron energies as a function of the CE phase. The laser parameters are the same as ones used in Fig. 1. The solid triangles are the original experimental data [22]. The experimental CE phase shifted by $0.15 \pi$ is also plotted (solid circles).

spectra [14], and high-resolution electron spectra in the crossed electric and magnetic fields [29]. To eliminate the boundary effect that the electron wave packet reaches the boundary and is bounced back unphysically, we split the wave function at a given time $t_{i}$ as

$$
\Psi\left(t_{i}\right)=\Psi\left(t_{i}\right)\left[1-F_{s}\left(R_{c}\right)\right]+\Psi\left(t_{i}\right) F_{s}\left(R_{c}\right)=\Psi_{I}\left(t_{i}\right)+\Psi_{I I}\left(t_{i}\right) .
$$

Here, $F_{s}\left(R_{c}\right)=1 /\left(1+e^{-\left(r-R_{c}\right) / \Delta}\right)$ is a split function that separates the whole space into the inner $\left(0 \rightarrow R_{c}\right)$ and outer $\left(R_{c}\right.$ $\left.\rightarrow R_{\text {max }}\right)$ regions smoothly. Here $\Delta$ represents the width of the crossover region. $\Psi_{I}$ represents the wave function in the inner region and it is propagated under the full Hamiltonian numerically. $\Psi_{I I}$ stands for the wave function in the outer region and it is propagated under the Volkov Hamiltonian analytically. We first calculate

$$
C\left(\mathbf{p}, t_{i}\right)=\int \Psi_{I I}\left(t_{i}\right) \frac{e^{-i\left[\mathbf{p}-\mathbf{A}\left(t_{i}\right)\right] \cdot \mathbf{r}}}{(2 \pi)^{3 / 2}} d^{3} \mathbf{r},
$$

then propagate $\Psi_{I I}$ from $t_{i}$ to the end of the pulse as

$$
\begin{aligned}
\Psi_{I I}\left(\infty, t_{i}\right) & =U_{v}\left(\infty, t_{i}\right) \int C\left(\mathbf{p}, t_{i}\right) \frac{e^{i \mathbf{p} \cdot \mathbf{r}}}{(2 \pi)^{3 / 2}} d^{3} \mathbf{p} \\
& =\int \bar{C}\left(\mathbf{p}, t_{i}\right) \frac{e^{i \mathbf{p} \cdot \mathbf{r}}}{(2 \pi)^{3 / 2}} d^{3} \mathbf{p},
\end{aligned}
$$

with $\bar{C}\left(\mathbf{p}, t_{i}\right)=U_{v}\left(\infty, t_{i}\right) C\left(\mathbf{p}, t_{i}\right)$. The final momentum distribution is obtained as

$$
\frac{d P(\mathbf{p})}{d E d \Omega}=\sqrt{2 E}\left|\sum_{i} \bar{C}\left(\mathbf{p}, t_{i}\right)\right|^{2},
$$

with $E$ the electron energy associated with p. We use the SFA in the outer region rather than in the whole region as 

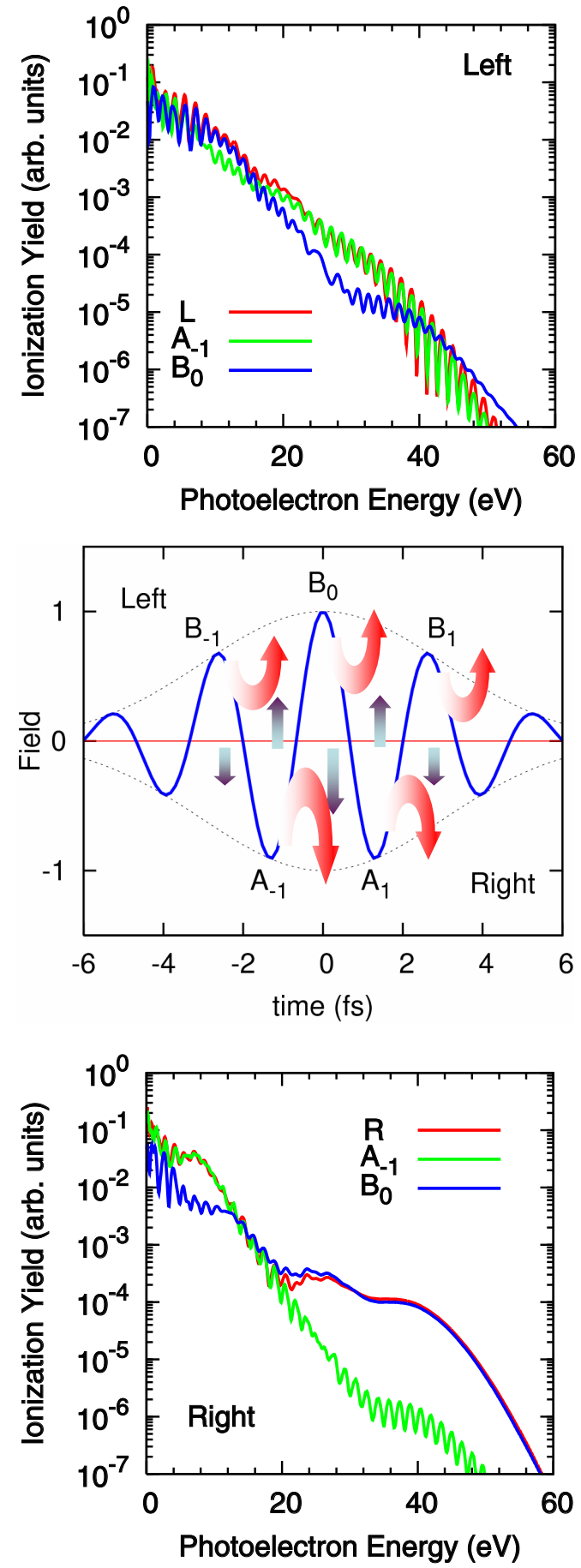

FIG. 3. (Color online) Simulated electron energy spectra from the right (right panel) and left (left panel) sides in the ultrashort intense laser field for $\delta=0$. The time-dependent laser field is depicted in the middle panel. The straight arrows stand for the direct ionization and the curved arrows stand for the scattering part. $A_{-1}$ and $B_{0}$ stand for the spectra without the ionization contribution from peak $A_{-1}$ or $B_{0}$. The laser parameters are the same as the ones used in Fig. 1.

done in the Lewenstein model [6]. As we know, in the outer region, the electron-parent-core interaction is much smaller than the electron-laser-field interaction and hence the propagation of the electron wave packet in the outer region can be calculated either numerically by Eq. (1) or analytically by Eq. (7).

To compare with the phase-dependent ATI experiment [22], we investigate the ATI spectra of Xe atoms in an ultrashort laser field. We use a model potential [30] to describe the valence electron $(5 p)$ interacting with the parent core. In the present simulation, we choose $R_{\max }=200$ a.u. and $R_{c}$ $=100$ a.u. We vary $R_{c}$ from 50 to 150 a.u. but the final results are insensitive to the choice of $R_{c}$ in this range. The key procedure is that we avoid the propagation of the wave packet in the outer region numerically. The outer region wave function is propagated by Eq. (7) so that there is no boundary problem any more.

Following the experiment [22], we present our simulated ATI momentum distribution by collecting the ATI yields on the right and left sides as

$$
\begin{aligned}
& \frac{d P_{R}(E)}{d E}=\int_{S_{R}} \frac{d P(\mathbf{p})}{d E d \Omega} d \Omega, \\
& \frac{d P_{L}(E)}{d E}=\int_{S_{L}} \frac{d P(\mathbf{p})}{d E d \Omega} d \Omega,
\end{aligned}
$$

where $S_{R}$ and $S_{L}$ stand for the integration over the right and left half spheres. Here the right (left) side means the negative (positive) electric field direction.

Figure 1 shows our simulated ATI spectra as a function of the CE phase. Overall we see that in the low-energy region $(E<10 \mathrm{eV})$, the ATI spectra from the right and left sides are of the same order and the ATI spectra are not sensitive to the CE phase. In the high-energy region $(E>40 \mathrm{eV})$, the ATI yields are very sensitive to the CE phase. As shown in the figure, the ATI yields from the right side are much larger than those from the left side for $\delta=0$. As we increase the CE phase from 0 to $0.6 \pi$, the difference decreases and finally the yields from both sides are close to each other. As the CE phase increases further, the high-energy ATI yields from the left side become larger than those from the right side as shown in Fig. 1(f). All the simulated results are in good agreement with the experimental results [22].

Figure 2 shows the ratio of ionization yields from the right and left sides at several photoelectron energies as a function of the CE phase. To compare with the experiment, the ratio of the total high-energy $(>20 \mathrm{eV})$ ATI yields is also plotted (solid line). The solid triangles are the original experimental data [22], which are in reasonable agreement with our simulated ones (solid line). If we shift the experimental CE phase by $0.15 \pi$ (solid circles), we find a better agreement between our simulations and the experimental ones. Thus the original experimental calibrated CE phase should be shifted by $0.15 \pi$. As shown in Fig. 1, the ionization yields also depend on the photoelectron energy. If we use the higherenergy ATI spectra as shown in Fig. 2 to calibrate the CE phase, we may get a more accurate CE phase.

The above discussion shows that we extract the CE phase more accurately by comparing our simulated ionization yields with the experimental ones. Next, we intend to depict the physical picture more clearly. For such a purpose, we rewrite Eq. (1) as 


$$
\begin{aligned}
\Psi(\infty) & =-i \sum_{n} \int_{t_{n-1}}^{t_{n}} U(\infty, \tau) V U_{0}(\tau,-\infty) \Phi_{0} d \tau+U_{0}(\infty,-\infty) \Phi_{0} \\
& =\sum_{n} \Psi_{n}(\infty)+U_{0}(\infty,-\infty) \Phi_{0} .
\end{aligned}
$$

Here, $\Psi_{n}(\infty)$ stands for the electron wave function that is created from $t_{n-1}$ to $t_{n}$ and then propagated under the full Hamiltonian to the end of the laser pulse. The final ATI spectra are obtained by summing over all $\Psi_{n}(\infty)$ contributions coherently. Let us study the dynamics by excluding the contribution from a given $\Psi_{n}(\infty)$.

Figure 3 shows the detailed ATI spectra on the right and left sides. We will first analyze the ATI spectra from the left side as shown in Fig. 3 (left panel). When we remove the contribution of $\Psi_{n}(\infty)$ that corresponds to the ionization from peak $A_{-1}$ (as depicted in the middle panel), the highenergy ATI yields do not change but the low-energy ATI yields drop. When we remove the contribution from peak $B_{0}$, the high-energy ATI (from 15 to $40 \mathrm{eV}$ ) yields drop almost by one order. At the high-energy end, the yields become larger than the original ones. From the above analysis we depict the following picture. The high-energy ATI electrons from the left side are ionized by the laser field at peak $B_{0}$, accelerated by the following peak $A_{1}$ or ionized by peak $B_{-1}$, and accelerated by peak $A_{-1}$, while the low-energy ATI electrons come from the direct ionization around peaks $A_{-1}$ and $A_{1}$. Such a physical picture can be illustrated more clearly from the right side ATI spectra as shown in Fig. 3 (right panel). When we remove the contribution from peak $A_{-1}$, the high-energy ATI yields drop dramatically, and hence we can state that the high-energy ATI electrons on the right side come from the ionization at peak $A_{-1}$ and are accelerated (or scattered) by peak $B_{0}$. Removing the contribution from peak $B_{0}$ does not affect the high-energy ATI spectra, but the lowenergy ATI yields drop significantly. By decomposing the contributions from each laser field peak, we see that the high-energy ATI electrons come from the ionization in one peak field, are bounced back and accelerated by the following laser peak, and recollide with the parent core. The lowenergy ATI electrons come from direct ionization, but the intermediate-energy ATI electrons come from both processes and the situation is more complicated. For an ultrashort pulse, there is only one big peak for $\delta=0$ so that the highenergy ATI spectra from the right side are structureless while the high-energy ATI spectra from the left side may be accelerated by peaks $A_{-1}$ or $A_{1}$, which have equal peak field strength. This results in the interference structure on the left side. All the analysis and observations are consistent with the rescattering model $[4,5]$ as well as the experimental observation [31]. Here we have illustrated the physical picture clearly from an $a b$ initio quantum simulation.

This research was partially supported by a Grant-in-Aid for Scientific Research (B) and (C) from the Japan Society for the Promotion of Science.
[1] X. F. Li, A. L'Huillier, M. Ferray, L. A. Lompre, and G. Mainfray, Phys. Rev. A 39, 5751 (1989).

[2] P. A. P. Salieres, A. L'Huillier, and M. Lewenstein, Adv. At., Mol., Opt. Phys. 41, 83 (1999).

[3] B. Walker, B. Sheehy, K. C. Kulander, and L. F. DiMauro, Phys. Rev. Lett. 77, 5031 (1996).

[4] P. B. Corkum, Phys. Rev. Lett. 71, 1994 (1993).

[5] K. C. Kulander and K. J. Schafer, in Proceedings of the Workshop on Super-Intense Laser Atom Physics (SILAP) III (Plenum Press, New York, 1993), p. 316.

[6] M. Lewenstein, P. Balcou, M. Y. Ivanov, A. L'Huillier, and P. B. Corkum, Phys. Rev. A 49, 2117 (1994).

[7] J. L. Krause, K. J. Schafer, and K. C. Kulander, Phys. Rev. A 45, 4998 (1992).

[8] C. J. Joachain, M. Dorr, and N. Kylstra, Adv. At., Mol., Opt. Phys. 42, 225 (1999).

[9] R. Wiehle, B. Witzel, H. Helm, and E. Cormier, Phys. Rev. A 67, 063405 (2003).

[10] S. Chelkowski, A. D. Bandrauk, and A. Apolonski, Phys. Rev. A 70, 013815 (2004).

[11] D. B. Milosevic, G. G. Paulus, and W. Becker, Phys. Rev. Lett. 89, 153001 (2002).

[12] D. B. Milosevic, G. G. Paulus, and W. Becker, Phys. Rev. A 71, 061404(R) (2005).

[13] A. Becker and F. H. M. Faisal, J. Phys. B 38, R1 (2005).

[14] M. Wickenhauser, X. M. Tong, and C. D. Lin, Phys. Rev. A
73, 011401(R) (2006).

[15] D. G. Arbo, S. Yoshida, E. Persson, K. I. Dimitriou, and J. Burgdorfer, Phys. Rev. Lett. 96, 143003 (2006).

[16] T. Brabec and F. Krausz, Rev. Mod. Phys. 72, 545 (2000).

[17] P. Agostini and L. F. DiMauro, Rep. Prog. Phys. 67, 813 (2004).

[18] C. M. Maharjan, A. S. Alnaser, I. Litvinyuk, P. Ranitovic, and C. L. Cocke, J. Phys. B 39, 1955 (2006).

[19] P. Salieres et al., Science 292, 902 (2001).

[20] J. Itatani et al., Nature (London) 432, 867 (2004).

[21] S. X. Hu and L. A. Collins, Phys. Rev. Lett. 94, 073004 (2005).

[22] G. G. Paulus et al., Phys. Rev. Lett. 91, 253004 (2003).

[23] A. Apolonski et al., Phys. Rev. Lett. 92, 073902 (2004).

[24] C. Lemell, X. M. Tong, F. Krausz, and J. Burgdorfer, Phys. Rev. Lett. 90, 076403 (2003).

[25] G. G. Paulus et al., Nature (London) 414, 182 (2001).

[26] D. B. Milosevic, G. G. Paulus, and W. Becker, Opt. Express 11, 1418 (2003).

[27] X. M. Tong and S. I. Chu, Chem. Phys. 217, 119 (1997).

[28] X. M. Tong and Shih-I. Chu, Phys. Rev. A 61, 021802(R) (2000).

[29] X. M. Tong and Shih-I. Chu, Phys. Rev. A 61, 031401(R) (2000).

[30] X. M. Tong and C. D. Lin, J. Phys. B 38, 2593 (2005).

[31] F. Grasbon et al., Phys. Rev. Lett. 91, 173003 (2003). 